\title{
Die Quellen des fünfsprachigen Wörterbuchs von Faust Vrančić
}

\author{
ISTVÁN VIG \\ ELTE BTK Szláv Filológiai Tanszék, H-1088 Budapest, Múzeum krt. 4/D. \\ E-mail: vigistvan@yahoo.com
}

(Received: 19 May 2015; accepted: 6 August 2015)

\begin{abstract}
Historical lexicography and etymology fail to examine the validity of words in old dictionaries. This gap is attempted to be filled up with the present study on the five-language dictionary (Latin-Italian-German-Croatian-Hungarian) published in 1595 and compiled by a Croatian author Faust Vrančić. In the paper, seven criteria are used. The analyzed words are equivalent to Latin nouns and adjectives. The Latin lexemes comprise more than half of the entries, which is a substantial sample to draw general conclusions. It is pointed out in the study that the dictionary provided help primarily in understanding Latin texts. It may have been to the greatest use of speakers of the four (non-Latin) vulgar languages with mother-tongue competence or those who were familiar with them. The dictionary seems not to have been adequate in all cases for those interested in these languages to enlarge their vocabulary.
\end{abstract} validity

Keywords: historical lexicography, five-language dictionary, etymology, Faust Vrančić,

1. Einführung. Zwei frühere Studien widmeten wir der Erforschung einiger Aspekte des fünfsprachigen (lateinisch-italienisch-deutsch-kroatisch-ungarischen) Wörterbuchs (1595) von Faust Vrančić. In der ersten Studie analysierten wir die Zuverlässigkeit des Dictionariums (VIG 2012). Es wurde festgestellt, dass das Wörterbuch an erster Stelle zum Verständnis lateinischer Texte geeignet war. Es war besonders für Muttersprachler der vier Vulgärsprachen bzw. für Leser nutzbar, die gute Kenntnisse dieser Sprachen besaßen. Auf Grund der schon erwähnten Fehler und Mängel war das Dictionarium eher weniger geeignet, die Wortkenntnisse der Benutzer, die keine Muttersprachler waren, zuverlässig zu erweitern. Diese Feststellungen mindern das Verdienst des Wörterbuchs nicht, besonders im Licht der Tatsache, dass noch Angaben hinsichtlich der Zuverlässigkeit anderer Wörterbücher des 16. Jahrhunderts und anderer Epochen fehlen. Auch in den Ausgaben von Calepinus von 1585 und 1586 befinden sich z. B. zahlreiche Fehler, obwohl die Äquivalente nicht von einer Person, sondern von mehreren Mitarbeitern eingetragen wurden (VIG 2012: 307).

Das Ziel der zweiten Studie war, die Sprachkenntnisse von Vrančić festzustellen (VIg 2014). Außer den Wortkenntnissen des Autors wollten wir auch seine Kompetenzen der grammatikalischen Regeln in den einzelnen Sprachen im Wörterbuch rekonstruieren. Es wurde festgestellt, dass Vrančić alle Sprachen in seinem Dictionarium gut beherrschte. Seine Wortkenntnisse hinsichtlich der einzelnen 
Vulgärsprachen weisen ausschließlich quantitative und keine qualitativen Unterschiede auf. Vrančićs Umschreibungen, seine eigenen Wortbildungen und auch seine sprachgeschichtlich-typologische Kompetenz der einzelnen Vulgärsprachen (VIG 2014: 285-287) beweisen gründliches Wissen der grammatischen Regeln.

Das Ziel der vorliegenden Studie ist es, die Quellen des Dictionariums definitiv zu bestimmen. Damit wird unsere originäre Zielsetzung vervollständigt, $d . h$. die Analyse von Vrančićs Werk aus drei eng zusammenhängenden Gesichtspunkten zu erforschen und auszuwerten.

2. Zusammenfassung der früheren Quellenforschungen und deren Methoden. Mit der Quellenforschung des Wörterbuchs von Vrančić beschäftigten sich ungarische und kroatische Forscher. Ihre Gedanken werden kurz, entsprechend der thematischen Eingliederung dargestellt.

In dieser Forschungsrichtung wurden konkret erwähnte Werke, nicht genau bestimmbare Quellen bzw. sprachliche Informatoren erwähnt. Was die Wörterbücher als Quellen der lateinischen, italienischen, deutschen und ungarischen Wörter betrifft, werden verschiedene Ausgaben des Wörterbuchs von Calepinus zitiert (s. Melich 1913: 242, Fokos 1924: 75-76, Dukat 1925: 107-108, Balázs 1975: 379-380, Sulyok 2001: 216). Als Quelle der ungarischen Lexeme wurde noch das Wörterbuchfragment von Gyöngyös (um 1560) (FoKOs 1924: 75-76) hinzugezogen. Für den deutschen Wortschatz wurden die Wörterbücher von Peter Cholinus, Johannes Frisius (1541), Josua Maaler (1561) als Quellen durch Žepić eingesehen (ŽEPIĆ 1992-1993: 567-577).

In Beziehung zu den deutschen Lexemen deutet Vladoje Dukat noch nicht identifizierte Wörterbücher an (DuKaT 1925: 107-108). Ljudevit Jonke geht vom Gebrauch von lateinischen, italienischen bzw. deutschen Quellen aus (JONKE 1992: 137), während Josip Vončina die Benutzung kroatischer Manuskripte annimmt, die zur Bibliothek von Anton Vrančić, dem Onkel unseres Autors, gehörten (VoNČINA 1979: 21-22).

Auch Beiträge von Informanten wurden angenommen: Mit deutschen und italienischen Helfern rechnet Dukat (s. Dukat 1925: 107-108), mit Deutschen Stanko Žepić (s. ŽEPIĆ 1992-1993: 576-577).

Bei der Identifizierung der Quellen sind die Prinzipien, auf denen das Verfahren beruht, von großer Bedeutung. Für die meisten Forscher haben die Wortübereinstimmungen, die Identität in der Schreibweise der Lexeme sowie die Abkürzung der Ausdrücke Beweiskraft. Unter den von Fokos erwähnten 14 Lexemen stimmen einige auch formell nicht, z. B. V.: Fenyüfa : GySz.: Lucz feniöfa, V.: tagitány [tágítani, inf.] : GySz.: meg oreghbitóm, Thagytom [megöregbítöm, tágítom, 1. Pers. Sing.] usw. (Fокоs 1924: 75-76). 32 mit dem Buchstaben „B“ beginnende Lexeme aus den Ausgaben von Calepinus aus dem Jahr 1564 bzw. 1605 [!] weisen mehrere lateinisch-italienische Übereinstimmungen auf, doch weniger in den lateinisch-deutschen Wortpaaren. Das veranlasst Dukat zu der Annahme, dass sich Vrančić gelegentlich auch eines anderen lateinisch-deutschen Wörterbuchs bediente (DukAT 1925: 107-108). Für János Balázs haben Abkürzungen wie z. B. Cal.: Sárbol valo edény [sárból való edény] $\rightarrow$ V.: Fold-edeny [földedény], Cal.: 
Iegnye farago áts [jegenyefaragó ács] $\rightarrow$ V.: Alch [álcs] Beweiskraft (BALÁzs 1975: 379-380). Diese Beweistypen nennen wir auf positiven Übereinstimmungen beruhende Beweise. $\mathrm{Zu}$ den Forschern, die sie anwenden, gehörte in einer ersten Phase János Melich und vermutlich auch Hedvig Sulyok (Melich 1906, SulyoK 2001). Melich betrachtete später falsche Äquivalente als Beweise der Quellennutzung (MeliCH 1913: 242). Zwei Autoren präzisieren ihre angewandte Methode bei der Quellenfeststellung nicht (JoNKE 1992, ŽEPIĆ 1992-1993).

Die Anzahl der in der Quellenforschung betrachteten Wörter ist sehr gering: nur eins bei Melich (1913), 12 bei Fokos, 32 bei Dukat, 17 bei Balázs, ohne Erwähnung bei Žepić und Sulyok.

Anhand der kurzen Zusammenfassung der Ergebnisse der Fachliteratur lassen sich die folgenden Bemerkungen feststellen. 1. Vier Wörterbücher wurden als Quellen in der Forschung identifiziert. 2. Unter ihnen ist Calepinus, außer für die kroatischen Wörter, die Quelle der Lexeme der anderen Sprachen. 3. Andere, nicht näher bestimmte Quellen werden in Bezug auf die lateinischen, italienischen, deutschen und kroatischen Wörter erwähnt. 4. Die Anzahl der Wörter, die die Quellennutzung beweisen, ist sehr gering. 5. Für die meisten Forscher gelten die positiven Übereinstimmungen als Quellennutzung von Vrančić. Kurz und einfach gesagt, heißt das, dass die Lexeme libro, Buch, knjiga, könyv, die als Äquivalente des lateinischen liber in einem früheren Wörterbuch vorkommen, den Gebrauch dieses Werks als Quelle beweisen. Diese Anschauung schließt die Annahme aus, dass gebildete Personen im Mittelalter und auch in späteren Perioden aktive Kenntnisse mehrerer Sprachen besaßen. Diese Anschauungsweise ist unserer Meinung nach die Rückprojizierung der Auffassung des sprachlich homogenen Nationalstaats des 19. und 20. Jahrhunderts. Die modernen Nationalstaaten strebten nach der Ausbildung homogener und einsprachiger Gesellschaften. Aber in früheren Perioden waren solche Bestrebungen nicht zu spüren. Das Lateinische war Jahrhunderte lang die Sprache der Wissenschaft und der internationalen Kommunikation von gebildeten Personen. Menschen, die mehrerer Fremdsprachen mächtig waren, bildeten keine Ausnahme. Der Ungar Márton Szepsi Csombor (1595-1622), der gute Kenntnisse der lateinischen und deutschen Sprache hatte, konnte problemlos 16171618 viele europäische Länder bereisen. Auch der Poet und Schriftsteller Miklós Zrínyi (1620-1664), Ban Kroatiens und Oberbefehlshaber der ungarischen Armee, beherrschte mehrere Sprachen. Er besaß in seiner Bibliothek italienische, ungarische, deutsche, spanische, französische, kroatische und niederländische Bücher. Die Annahme, dass er keine aktiven Kenntnisse der kroatischen, ungarischen, lateinischen, italienischen und deutschen Sprache besaß, ist auszuschließen. Noch andere Beispiele könnten als Beweis erwähnt werden, dass die Kenntnisse mehrerer Fremdsprachen keine Rarität bei ausgebildeten Personen des 16. und 17. Jahrhunderts waren. Ebenso ist anzunehmen, dass auch der nicht weniger ausgebildete Vrančić aktiv der Sprachen seines Wörterbuchs mächtig war.

3. Interpretation der lateinischen Stichwörter und deren Äquivalente. Als Voraussetzung der Quellenforschung wurden die lateinischen Stichwörter und deren Äquivalente semantisch präzise interpretiert. Das Verfahren umfasste mehrere 
Stufen. Zuerst wurde die Bedeutung der lateinischen Lexeme anhand der modernen zweisprachigen Wörterbücher (lateinisch-italienisch, lateinisch-deutsch, lateinischungarisch) festgestellt (DILL, GYLM, LGSWLD, FLNySz).

Dabei war die Tatsache zu berücksichtigen, dass Lexeme bzw. Bedeutungen der mittelalterlichen Latinität unter den lateinischen Stichwörtern des Wörterbuchs vorkommen konnten. Um sie zu identifizieren, wurden auch Wörterbücher benutzt, die den mittellateinischen Wortschatz Böhmens, Frankreichs, der Niederlande, Kroatiens, Polens und Ungarns bearbeiten (Du CANGE, GMILRH, LLMAH, LLMAI, LLNMA, LMALB, LMILP).

Bei der Interpretation der lateinischen Stichwörter durch heutige Äquivalente werden wir mit großer Vorsicht vorgehen. Erstens war zu beachten, dass die heutigen Sprachen eine reichere Lexik bzw. andere Wortbedeutungen haben als die Sprachstufen früherer Perioden. Zweitens war zu berücksichtigen, dass viele Wörter untergingen bzw. die Bedeutungen von vielen Lexemen verloren gingen. Bei der Kontrolle der Äquivalente bei Vrančić wäre es falsch, solche Wörter als nicht richtig zu interpretieren, die in den modernen Wörterbüchern keinen Niederschlag gefunden haben. Für die Kontrolle der Äquivalente bei Vrančić mussten wir allen Lexemen nachgehen, die wesentlich von den heutigen Äquivalenten abweichen. Deswegen wurden auch sprachhistorische bzw. dialektale Wörterbücher hinzugezogen.

Für diese Kontrolle der Wörter bzw. deren Bedeutungen in der deutschen und italienischen Sprache wurden sprachhistorische Lexika benutzt, die ein sehr reiches Sprachmaterial bieten (GDLI, DWB). In Beziehung zur deutschen Wortgeschichte erwiesen sich die Wörterbücher der mittelhochdeutschen bzw. der frühneuhochdeutschen Periode (MHDHWB, FNHD) als nützlich. Für die Feststellung des ungarischen Wortschatzes und dessen Bedeutungen wurden mehrere Werke benutzt (TESz, RMG, SzT). Auch die Daten des ungarischen sprachhistorischen Wörterbuchs $(\mathrm{NySz})$ und des Urkundenwörterbuchs $(\mathrm{OklSz})$ wurden mit dem nötigen Vorbehalt betrachtet. Beim NySz ist, auf Grund seiner auf vorherigen Wörterbüchern (z. B. dem Wörterbuch von Albert Szenci Molnár) beruhenden Definitionen, die Unzuverlässigkeit nicht auszuschließen.

Für eine genauere Kontrolle der Wörter und deren Bedeutungen wurden auch Glossare eingesehen, die als Anhang moderne Ausgaben einiger Werke aus dem 16. und 17. Jahrhundert ergänzen: Gáspár Heltai (Heltai 1981), Mihály Bethlen (Bethlen 1981), Albert Szenci Molnár (Szenci Molnár 1976), Bálint Balassa (JAKAB-BÖLCSKEI 2000), Pál Tállyay (IsTVÁNFFY 2001), István Gyöngyösi (GYÖNGYÖSI 1998, 1999, 2000, 2002, 2003).

Hinsichtlich des italienischen Sprachraums wurden die Wörterbücher der venezianischen Mundart, der Dialekte Veneziens und Dalmatiens (AIS, DDF, DDV, DSFEDC, EV, GDDT, VDCh, VDVD, VFDB, VG) betrachtet.

Im deutschen Sprachraum erwies sich das Wörterbuch der schwäbischen Dialekte (FISCHER 1904-1914) als nützlich.

Im Bereich des ungarischen Sprachraums wurden die Daten des regionalen Wörterbuchs der ungarischen Sprache (ÚMTSz) benutzt. 
4. Die angewandten Methoden unserer Quellenforschung. Wie oben bereits erwähnt, ist es nicht begründet anzunehmen, dass Vrančić nicht der Sprachen seines Dictionariums mächtig war. Es ist auch nicht auszuschließen, dass er in bestimmten Fällen Quellen benutzte. Für die Feststellung der Quellen von Vrančić wandten wir das Prinzip der negativen Übereinstimmungen an. Dazu gehören die nicht bewiesenen Wörter bzw. Wortformen, die semantisch falschen Äquivalente: Ihr Vorkommen weist in zwei Werken einen engen Kontakt auf. Orthografische Merkmale wurden außer Acht gelassen, weil keine der modernen Sprachen des Wörterbuchs am Ende des 16. Jahrhunderts feste Rechtsschreibnormen hatte.

Um unsere Zielsetzungen auszuführen, wurden die Substantive und Adjektive in vier Sprachen im Wörterbuch analysiert. Diese Wahl wurde durch die Tatsache motiviert, dass die Gesamtzahl der Substantive und Adjektive (inklusive der Partizipien) mehr als die Hälfte der Lexeme (3462 gegenüber 5467 Einheiten) im Dictionarium ausmacht. Abgesehen von den Adverbien, Konjunktionen und Zahlwörtern überschreitet die Anzahl der Substantive und Adjektive die der Verben. Deswegen genügt diese Wortmenge, um allgemeine Schlussfolgerungen zu ziehen.

5. Die erforschte Textvariante. Für die Analyse wurde die zweite FaksimileAusgabe des Wörterbuchs von Vrančić (1992) benutzt. Sie bildete auch den Gegenstand der Forschung kroatischer Gelehrter.

Unter den möglichen Quellen wurde zuerst die Rolle des Wörterbuchs von Calepinus festgestellt. Auch andere Werke wurden als mögliche Quellen in Betracht gezogen. Hinsichtlich des ungarischen Wortschatzes wurden das dreisprachige Wörterbuch (lateinisch-deutsch-ungarisch) von Joannis Murmellius (1533), das fünfsprachige Wörterbuch (lateinisch-italienisch-französisch-tschechisch-ungarisch) von Gábor Pesti (1538), das Wörterbuchfragment von Gyöngyös (um 1560, dessen viele kopierte Exemplare im Verkehr sein konnten) und das lateinisch-ungarische Wörterbuch von Szikszai Fabricius konsultiert (SzIKSZAI FABRICIUS 1590). Ihre Identifizierung fand mit Hilfe der falschen Äquivalente in den alten Glossaren Ungarns (RMG) statt.

In Beziehung zum italienischen Wortschatz zogen wir nur Calepinus in Betracht. Diese Entscheidung lässt sich durch verschiedene Gründe erklären. Die mehrsprachigen Calepinus-Wörterbücher genossen große Popularität. Das zehnsprachige Calepinus-Wörterbuch schien bei erster Annäherung eine mögliche Quelle für Vrančić zu sein. Sein lateinischer, italienischer und deutscher Wortschatz war eine potenzielle und leicht verwendbare Basis für Vrančić. Andere lateinisch-italienische lexikalische Werke wurden nicht in Betracht gezogen, weil sie in Ungarn nicht zugänglich sind.

Was den deutschen Wortschatz betrifft, wurde das lateinisch-deutsche Wörterbuch von Petrus Dasypodius (1536) als wichtiges Werk betrachtet. Es hat eine wichtige Rolle in der Geschichte der lateinisch-deutschen Wörterbücher (DE SMET 1974). Obwohl das Wörterbuch von Dasypodius ein sehr vertrauenswürdiges Werk ist, ist es nicht auszuschließen, dass es in eigenen Fällen falsche Äquivalente beinhaltet, die auch von späteren Wörterbüchern übernommen wurden. Auf Grund dieser Betrachtungen ist es fraglich, ob das Wörterbuch von Josua Maaler (1561) 
eine Quelle des deutschen Wortschatzes von Vrančić sein konnte. Die falschen Übereinstimmungen mit Dasypodius weisen darauf hin, dass die Urquelle das Wörterbuch von Dasypodius ist. Die Daten aus der Zeit nach Dasypodius wurden anhand der Daten des DWB ausgewertet. Hinsichtlich des deutschen Wortschatzes benutzten wir auch die Daten von Calepinus aus den Jahren 1585 und 1586.

Hinsichtlich der kroatischen Lexeme wurden die Daten des viersprachigen Wörterbuchs (deutsch-lateinisch-slowenisch-italienisch) von Hieronymus Megiser (1592) eingesehen. Im Wörterbuch sind etwa 274 kroatische Wörter durch das Kennzeichen „Cr“ markiert. Es verweist auf kajkawische Wörter, die viele Ähnlichkeiten mit slowenischen Wörtern haben. Demgegenüber gibt es aber siebzig Wörter, die nur in den čakawischen bzw. štokawischen Mundarten bewiesen sind (VIG 2005: 267-272).

6. Der Vergleich des Wörterbuchs von Calepinus und des Dictionariums. Melich war der Meinung, dass die Ausgabe von Calepinus aus dem Jahr 1586 als Quelle des Dictionariums diente. Eine genauere Betrachtung von Melichs Anschauung führt zu folgenden Fragestellungen: rück?

1) Gehen die lateinischen Stichwörter des Dictionariums auf Calepinus zu-

2) Ist ein Beispiel ausreichend für die Feststellung, dass Calepinus die Hauptquelle (angeblich nur der ungarischen Wörter) des Wörterbuchs von Vrančić war.

3) In welchem Maß diente Calepinus als Quelle für die ungarischen Lexeme? Welche Wörter wurden von Vrančić übernommen und welche nicht?

4) Außerdem stellt sich die Frage, ob sich Vrančić auch für die italienischen bzw. deutschen Äquivalente bei Calepinus bediente oder nicht?

Um diese Fragen zu beantworten, wäre die einfachste Lösung gewesen, alle italienischen, deutschen und ungarischen Äquivalente der lateinischen Stichwörter des Dictionariums mit Calepinus zu vergleichen. Um dieses Verfahren einfacher und gleichzeitig effizienter zu machen - die Kontrolle der insgesamt etwa 15000 italienischen, deutschen und ungarischen Äquivalente und der ungefähr 5000 lateinischen Stichwörter hätte zu viel Zeit und Mühen gebraucht -, schien es uns zweckmäßiger, solche Wörtergruppen auszuwählen, deren Analyse begründete Antworten auf die Fragen geben konnte. Daher wurden die folgenden Wortgruppen ausgewählt:

1) Die lateinischen Stichwörter beider Wörterbücher. Ihr Vergleich erwies sich als wichtig für die endgültige Feststellung der Abhängigkeit der lateinischen Stichwörter des Dictionariums von Calepinus. Dies ist der Ausgangspunkt für die Analyse der Äquivalente.

2) Aus dem Alten Ungarischen Glossar (RMG) wurden solche lateinisch-ungarischen Wortpaare exzerpiert, die sich nur in Vrančićs Dictionarium befinden. Es ist auszuschließen, dass sie nicht von Vrančić aus anderen Wörterbüchern und Glossaren übernommen wurden. Diese Lexeme, die wir der Einfachheit halber Vrančićs autonome ungarische Äquivalente nennen, lassen sich in zwei Gruppen gliedern. Zur ersten Gruppe gehören die lateinisch-ungarischen Wortpaare, die nur bei Vrančić belegt sind. Die zweite Gruppe wird von lateinisch-ungarischen 
Wortpaaren gebildet, deren lateinische Elemente auch in früheren Werken belegt sind. Ihre ungarischen Äquivalente kommen aber nur im Dictionarium vor. Anhand der autonomen ungarischen Ausdrücke wurden auch die italienischen und deutschen Äquivalente bei Vrančić mit denen bei Calepinus verglichen.

Obwohl wir die traditionellen positiven Übereinstimmungen (auf formellen Übereinstimmungen bzw. Ähnlichkeiten beruhend) als Beweiskraft nicht akzeptieren, wandten wir dieses Prinzip trotzdem an, um nach diesem Prinzip eventuelle Quellen des Dictionariums festzustellen.

Der Vergleich der lateinischen Stichwörter beider Wörterbücher erbringt, dass viele Stichwörter bei Vrančić nicht bei Calepinus belegt sind. Diese sind die Folgenden: abominatio, arefieri, attavia, bilis, binis, breviter, buccella, campana, capito, captus, citatus, citare, citus, computus, desponsatio, dirrimere, divisus, divitiae, fagistiare, falcare, famere, februarius, firmitas, foratus, forter, gestus, gratitudo, honorandus, iamdiu, Ister, latare, leprosus, libenter, lucidare, nasu, natatis, nudipes, nutus, obligatus, oriens, parcus, perpexus, pinguefacere, pletique, prodigalitas, pulsus, quamvis, quoquomodo, quorsum, rapina, restio, sanctificare, sexdecim, tepefacere, venustus, volatio, usuvenit.

Diese Wortliste lässt zwei Schlussfolgerungen zu:

1) Vrančić bediente sich nicht bei Calepinus für die Zusammenstellung der lateinischen Stichwörter.

2) Er benutzte Calepinus frei und autonom. Er übernahm bestimmte Wörter, die ihm für seine Zwecke wichtig waren.

Unserer Meinung nach ist die erste Annahme richtig: Im umgekehrten Fall wäre es für ihn einfacher gewesen, die lateinischen Stichwörter von Calepinus einfach zu übernehmen.

7. Unterschiede der lateinischen Stichwörter bei Calepinus bzw. Vrančić. Das Dictionarium von Vrančić hat 53 lateinische Wörter, die formell nicht mit denen von Calepinus übereinstimmen. Die Abweichungen weisen orthografische (z. B. cuppa : cupa) und morphologische (gracus : graculus) Merkmale auf. Außerdem sind viele Substantive in Pluralform bei Calepinus belegt, die bei Vrančić (z. B. histriones : histrio) in Singularform vorkommen. Die Annahme, dass die positiven Übereinstimmungen zwischen vermuteten Quellen und dem Dictionarium von Vrančić ein Beweis des Quellengebrauchs sind, wird durch die erwähnten Beispiele nicht unterstützt. In diesem Sinn gilt Calepinus nicht als Quelle der lateinischen Stichwörter des Dictionariums. Warum sollte Vrančić die Stichwörter von Calepinus verändern? Ein Platzmangel im Wörterbuch ist auszuschließen.

Die autonomen ungarischen Äquivalente von Vrančić sind teilweise identisch mit den Äquivalenten bei Calepinus und teilweise unterschiedlich. Es wurde schon erwähnt, dass auch die italienischen und deutschen Äquivalente in unserer Analyse in Betracht gezogen wurden.

Zur Feststellung der Übereinstimmungen bzw. der Unterschiede wurden auch in diesem Fall die positiven Übereinstimmungen, d. h. die vollständige morphologische Übereinstimmung der Wörter, betrachtet. Die Äquivalente bei Vrančić ähneln häufig den Stichwörtern bei Calepinus, aber nicht völlig. Als Illustration 
werden einige Wortpaare erwähnt, die auf andere Beispiele im Dictionarium hinweisen. Vor dem Doppelpunkt befinden sich die Wörter von Vrančić, nach dem Doppelpunkt sind die Äquivalente von Calepinus zitiert: irigység : irigyködés (s. v. aemulatio), hasonlétani : öszvehasonlitom (s. v. comparare), illet : megilletöm (s. v. contingere), megkent : megkenetött (s. v. delibutus), rohanás : berohanás (s. v. irruptio), iszonyú : iszonyú latorság (s. v. nefas). Nach der Logik von János Balázs könnte man folgendermaßen argumentieren: Vrančić übernahm die Wörter von Calepinus mit bestimmten Veränderungen. Die Verbalpräfixe bzw. die Substantive in der Konstruktion Adjektiv + Substantiv wurden weggelassen und auch die Partizipien wurden verändert. Eine solche Hypothese wäre übrigens ein eklatanter Beweis der reinen Grundkenntnisse von Vrančić in Bezug auf die Sprach- und Wortbildungsregeln der ungarischen Sprache. Es stellt sich aber auch die Frage: Wenn Vrančić die erwähnten Wörter nicht kannte, warum übernahm er sie mit Veränderungen? Der Einwand des Platzmangels ist auszuschließen: In der Spalte der ungarischen Äquivalente steht ein Platz für 14-17 Buchstaben zur Verfügung, vgl. z. B. gradich, laitoria (17 n, s. v. scala) und meczenni-uagni (14 n, s. v. secare). Die deutschen Äquivalente von sedare (stillen, milteren) nehmen $18 \mathrm{n}$ ein. Die ungarischen Wörter irigykedés, meggillet/megilletni konnten auch problemlos eingeführt werden. Auch das Verb öszvehasonlétani (16 n) konnte leicht benutzt werden, besonders im Licht eines kürzeren Belegs im Calepinus: összevetöm (10 n), dessen Infinitiv (összevetni, Vrančić zitiert die Verben im Infinitiv) ebenso lang ist. Das oben Gesagte gilt auch für den Ausdruck iszonyú latorság (16 n). Außerdem ist noch zu betonen, dass zahlreiche lange Ausdrücke in der deutschen Spalte häufig getrennt (z. B. widerspennig-sein) bzw. abgekürzt (geinen, aufgině, s. v. hiare) werden.

Diese Argumentation ist geeignet aufzuweisen, dass Vrančić die Wörter des Dictionariums nicht in der Druckerei, am Tisch des Setzers zusammenfasste. Es ist auch wenig wahrscheinlich, dass er sich schon während der Herstellung seines Manuskriptes des Formats des Wörterbuchs bewusst war. Andererseits ist anzunehmen, dass er lange Erklärungen, die mehrere Reihen beanspruchten, vermeiden wollte: Ein solches Verfahren hätte der Benutzung und der Übersichtlichkeit des Wörterbuchs geschadet. Auch in den modernen mehrsprachigen thematischen Wörterbüchern befinden sich tendenziell die Äquivalente in der gleichen Spalte wie die Stichwörter. Nur in einigen Fällen nehmen die Äquivalente mehr als eine Reihe ein, was von der Anzahl der Wörter und deren Umfang abhängig ist. ${ }^{1}$

Die erwähnten Argumente weisen noch auf eine wichtige Bemerkung hin. Die formellen Ähnlichkeiten zwischen Calepinus und Vrančić beweisen die Benutzung von Calepinus als Quelle nicht. Eine solche Vermutung erweist sich als reine Spekulation, die durch keinen Beweis unterstützt wird. Unter den autonomen ungarischen Äquivalenten von Vrančić stimmen insgesamt zwölf Wörter mit den

\footnotetext{
${ }^{1}$ Vgl. das sechssprachige Konferenzwörterbuch: HERBERT 1976. In der deutschen Spalte kommen häufig Äquivalente vor, die sich auf zwei bzw. drei Reihen ausdehnen: die Türhüter, die Platzanweiser, die Saaldiener (HERBERT 1976: 63); die Ordnung, das Reglement (HERBERT 1976: 27). 
Äquivalenten bei Calepinus überein: nem hasonló (s. v. absimil), felherpenteni (s. v. absorbere), kosár (s. v. corbis), hív (s. v. fidus), világos (s. v. illustris), tréfa (s. v. iocus), gyertya (s. v. lucerna), rab (s. v. mancipium), ködös (s. v. nebulosus), megfeddeni (s. v. obiurgare), jutalom (s. v. praemium), karó (s. v. statumen). In sieben Fällen kommt bei Vrančić auch ein anderes Äquivalent vor, das bei Calepinus nicht belegt ist. Dies beweist, dass Vrančić die ungarischen Äquivalente der lateinischen Stichwörter kannte. Er brauchte daher nicht den Calepinus, um noch ein weiteres ungarisches Wort in sein Wörterbuch hinzufügen. Er kannte auch die ungarischen Äquivalente der anderen fünf lateinischen Wörter: Die Übereinstimmung seiner Wörter mit den Äquivalenten von Calepinus lässt sich als Zufall erklären.

Diese Feststellung in Bezug auf die ungarischen Äquivalente gilt auch für die italienischen bzw. deutschen Äquivalente, obwohl die Anzahl der übereinstimmenden Wörter mit den Lexemen von Calepinus höher ist. Aber 156 italienische und 165 deutsche Ausdrücke unterscheiden sich von den Äquivalenten bei Calepinus. Sie machen mehr als ein Drittel der Äquivalente (italienisch $32 \%$, deutsch $38 \%$ ) von 409 lateinischen Stichwörtern aus. Es ist auszuschließen, dass ihre Quelle Calepinus ist.

Neun lateinischen Stichwörtern entsprechen je zwei deutsche Äquivalente, unter denen eins nicht bei Calepinus belegt ist. Es sind die Folgenden: Scheltwort (s. v. contumelia), $\operatorname{Kampf}$ (s. v. dimicatio), Licht (s. v. lucerna), geküglet (s. v. orbicolatus), Zirkel (s. v. orbis), begehren (s. v. precari), erhacken (s. v. suavis), Stab (s. v. scipio), unerständig (s. v. stolidus), sanft (s. v. suavis), Gwalt (s. v. vis). Diese Beispiele beweisen, ähnlich den ungarischen Äquivalenten, dass Vrančić Calepinus als Quelle nicht brauchte.

Die Analyse der italienischen Äquivalente von Vrančić, die mit denen bei Calepinus übereinstimmen, zeigt, dass 124 lateinischen Stichwörtern mehrere Äquivalente entsprechen. Meistens sind es zwei, manchmal drei und in einigen Fällen mehrere Lexeme. Bei der Annahme, dass Vrančić die betreffenden italienischen Äquivalente unbekannt gewesen wären, würde man die Übernahme des ersten Lexems aus dem Calepinus erwarten. Die Situation ist aber anders: In 77 Fällen stimmen die Lexeme von Vrančić mit den ersten Äquivalenten bei Calepinus überein. 41 Wörter entsprechen den zweiten Ausdrücken bei Calepinus, in vielen Fällen dem dritten und zweimal dem vierten Lexem bei Calepinus. Diese „Übernahmen“ lassen vermuten, dass Vrančić die Äquivalente bei Calepinus kannte und sie aus einem bestimmten Grund auswählte. Diese Auswahl unter den Äquivalenten ist ein Beweis der Wortkenntnisse unseres Autors und bedeutet, dass er nicht Calepinus benutzte.

Die deutschen Äquivalente im Dictionarium entsprechen den ersten bzw. zweiten und dritten Lexemen von Calepinus. In 104 Fällen stimmen sie mit den ersten Lexemen bei Calepinus überein. 55 Wörter entsprechen den zweiten Äquivalenten bei Calepinus und sieben Lexeme den dritten. Man kann nicht beweisen, ähnlich wie im Fall der italienischen Äquivalente, dass Calepinus als Quelle der deutschen Lexeme diente. 
Es ist klar geworden, dass die positiven Übereinstimmungen die Benutzung von Calepinus als Quelle nicht beweisen. Als Methode bleibt die Anwendung der negativen Übereinstimmungen, um die Beziehung zwischen dem Dictionarium und den möglichen Quellen festzustellen. Es wurde schon erwähnt, dass dazu die nicht belegten fehlerhaften Wörter bzw. Wortformen und die falschen semantischen Entsprechungen gehören, die in zwei Werken identisch sind. Sie haben ausschlaggebende Beweiskraft. Die orthografischen Übereinstimmungen wurden nicht in Betracht gezogen, weil noch keine gemeinsamen Rechtschreibregeln im 16. Jahrhundert existierten. Die deutschen Interpretationen der lateinischen Stichwörter beruhen auf den Daten von LGSWLD. Denen folgen die Lexeme von Vrančić. Nach dem Doppelpunkt stehen die Wörter der Quelle von Vrančić.

\section{Die effektiven Quellen des Dictionariums}

\subsection{Die Wörterbücher von Calepinus (1585, 1586)}

\subsubsection{Italienische Äquivalente}

1. aqualiculus 'Trog, Tränke' albio : albio, albuolo da porci. Die Bedeutung 'Schaff, Gefäß' von albio ist nicht aus dem Italienischen belegt (GDLI 1: 291).

2. cothurnus '1. Jagdstiefel. 2. Bühnenschuh des tragischen Schauspielers' bolzachino : borzachino, coturno. Das Wort ist nicht in der Wortgeschichte der italienischen Sprache belegt. Es kommt in den Auflagen aus 1549, 1585 und 1586 von Calepinus vor: borzacchini Pl. (1549), borzachino (1585, 1586). Es geht um ein in Dokumenten nicht belegtes Wort, das in den verschiedenen Auflagen belegt ist. Der Graphemwechsel $r \rightarrow l$ ist ein Druckfehler.

3. caritas 'Teuerung' carestia : carestia, inopia. Das italienische Substantiv carestia heißt 'Warenmangel' (vgl. DELIN: 299).

4. clades 'Schaden, Unglück, Unheil' uccisione: calamità, uccisione.

5. coagulum 'das Lab' cagliarino : cagliarino, coagolo, conaglio. Die heutigen italienischen Äquivalente sind coagulo/coagolo bzw. caglio (DILL: 245). Das Wort cagliarino ist nicht belegt (vgl. GDLI 2: 505). Das Äquivalent von Vrančić stimmt mit dem Wort auf dem ersten Platz von Calepinus überein, das nicht einmal in der italienischen Wortgeschichte belegt ist. Außerdem fand eine Vertauschung von zwei Buchstaben statt.

6. defectio 'das Abnehmen, Schwinden, Schwächung' debolezze : debollezze. Das italienische Wort heißt 'Schwäche, Kraftlosigkeit'.

7. fimbria 'Franse, Troddel' le bande : le bande, le frange. Die Pluralform le bande bedeutet 'die Seite von etw.'

8. glomus 'Knäuel' giemo : gomitolo, ghiomo, gemo, gomiscello. Giemo ist in der italienischen Wortgeschichte unbekannt. Der Unterschied zwischen giemo bzw. gemo ist nur scheinbar, weil der Buchstabe «ii in der Aussprache keine Rolle spielt.

9. grassator 'Wegelagerer, Bandit' assassino : malandrino, ladrone, assasino da strada. Vrančić übernahm das erste Element der semantisch richtigen Umschreibung 'Mörder an der Landstraße', das so allein belegt nur 'Mörder' heißt. 
10. ignavia 'Trägheit, Lässigkeit, Mangel an Energie' dappoccaggine : paura, pigritia, dapoccagine. Dapoccaggine bedeutet '1. Ungeschicklichkeit. 2. Schwäche, Lumpigkeit'.

11. ilia '1. die Weichen. 2. Eingeweide, Magen' $i$ fianchi : $i$ fianchi. I fianchi bedeuten '1. Hüfte. 2. die Seite von etw.'

12. marcor '1. Welkheit. 2. (übertragener Sinn) Untätigkeit' marcia : marcia (vgl. marcia, marcito [1586]). Das Substantiv marcia ist wegen seiner Bedeutung ('Eiter') semantisch falsch. Auch die anderen Äquivalente des Dictionariums: ted. Muglose, kroat. gnjilad, ung. penisz erweisen sich als falsch, obwohl sie nicht auf Calepinus zurückgehen.

13. persica 'Pfirsich' persichi ( $\mathrm{Pl}$.) : resco, persico. Persico bedeutet 'Pfirsichbaum'. Das erste Äquivalent von Calepinus lässt sich nicht identifizieren.

14. protervia 'Tollkühnheit, Frechheit, Keckheit' lascivia : soperbia [!], sfacciatagine, ribalderia, lascivia, ostinatione, arrogantia. Vrančić übernahm eines der semantisch falschen Äquivalente von Calepinus 'Ausgelassenheit, Zügellosigkeit, Geilheit'.

15. scabellum 'Schemel, Fußbank' banchetto : banchetta, scabello, deschetto. Der Unterschied zwischen Vrančić und Calepinus liegt darin, dass Ersterer eine Diminutivform aus banchetto 'Bank' benutzt, während bei Calepinus die Diminutivform aus banca 'dies.' belegt ist. Das italienische Äquivalent des lateinischen Stichwortes ist sgabello (DILL: 1410), das in der Variante scabello seit dem 14. Jahrhundert, als sgabello seit 1550 belegt ist. Es ist nicht auszuschließen, dass die Formen mit/g-/ im Anlaut in früheren Perioden entstanden (DELIN: 1519). Auch das ungarische Äquivalent von Vrančić erweist sich als semantisch falsch, aber es geht nicht auf Calepinus zurück.

16. spatium '1. Raum, Ausdehnung, Weite, Größe. 2. Zeitraum, Zeit, Dauer, Frist, Gelegenheit (für etw.)' aggio : spatio, aggio, tiempo. Das Substantiv aggio bedeutet ' 1 . aus einer im Auftrag eingenommenen Summe auf Prozent, Prämie. 2. Agio'.

17. subula 'Pfriem, Ahle' lina: lina o lesina, over subli di calegaro. Unter den Äquivalenten von Calepinus ist nur lesina in der italienischen Wortgeschichte bekannt. Sublia lässt sich entweder als Druckfehler von subula interpretieren oder als ein aus subula gebildetes Wort, dessen Ergänzung ein Substantiv der venezianischen Mundart enthält (caligaro).

18. suspendium 'Erhängung' appiccamento : appicamento 'Aufhängung, Daranhängung (eines Gegenstandes)'.

19. tabes 'Fäulnis, Verwesung' tisico : tisichezza, mal di tisico, marcia, sangue marcio. Tisico als Adjektiv bedeutet 'lungenkrank', als Substantiv 'Lungenkrankheit'. Vrančić übernahm ein Element des Ausdrucks von Calepinus, das eigentlich 'Krankheit der Lunge' bedeutet.

20. vepres 'Dornstrauch' spine : spine (Pl.) 'Stachel, Dorn'. Unter den heutigen Äquivalenten des lateinischen Stichwortes ist spino '1. dornige Pflanze. 2. Dornstrauch' neben pruno, cespuglio spinoso, roveto, macchia belegt (DILL: 1673). Das falsche Äquivalent bei Calepinus ist entweder auf die Unsicherheit des Ver- 
fassers des Wortartikels oder den Fehler des Druckers zurückzuführen. Das ebenfalls falsche ungarische Äquivalent - bojtorján - von Vrančić ist bei Calepinus nicht belegt.

21. vocabulum 'Name' dittione [dizione] : dittione, vocabulo, voce. Das einzige semantisch falsche Äquivalent von Calepinus '1. Aussprache. 2. das Vortragen, Vortragsart. 3. Sprache, Stil’ wurde von Vrančić übernommen.

\subsubsection{Deutsche Äquivalente ${ }^{2}$}

1. aedacitas 'Gefräßigkeit' fraesserey : Essigkeit oder fraßerey.

2. crapula 'Rausch, Katzenjammer' frasserey : das hauptwee von dem prassen: item, Trunckenheit, fraesserey, füllerey. Auch das kroatische Äquivalent von crapula erweist sich als semantisch falsch: Žarstvo bedeutet 'Fresserei'.

3. culex 'Mücke, Schnake' bram : ein mugg oder braem. Bremse zeichnet ein anderes Denotatum.

4. fastus 'Dünkel, Stolz, Hochmut' pracht : pracht, stolzheit, hochmut. Es fehlen Belege der Bedeutung 'Dünkel, Stolz, Hochmut' von Pracht in der deutschen Wortgeschichte.

5. gigas 'Gigant' held : ein ryss oder held. Die Bedeutung 'Gigant' von Held ist in der Wortgeschichte der deutschen Sprache nicht belegt. Auch das kroatische Äquivalent von gigas, vukodlak 'Vampir, Monster' ist semantisch falsch.

6. induciae 'Waffenstillstand' aufzug : aufzug: ein anstand oder auffzug des krieges. Die Umschreibung von Calepinus ('Aufschiebung des Krieges') lässt sich als Entsprechung der Bedeutung des lateinischen Stichwortes interpretieren. Aber die bloße Übernahme des Kopfes des Ausdrucks ohne seine Ergänzung erweist sich semantisch als falsch. In der deutschen Wortgeschichte ist das Substantiv Waffenstillstand seit dem 18. Jahrhundert belegt. Frühere Belege für das Denotat sind Stillstand bzw. Anstand (DWB 1: 786; DWB 13: 319). Obwohl das Letztere bei Calepinus belegt ist, wurde es von Vrančić nicht übernommen. Auch das kroatische Äquivalent des Stichwortes ist semantisch falsch: Prodljenje [produljenje] bedeutet ' 1 . Verlängerung. 2. Fortsetzung'.

7. mancipium 'Kaufsklave, Sklave' leibaigner : ein leibegner mensch, als da sind die man erkaufft im krieg fahet. Aus der Paraphrase wurde das Adjektiv von Vrančić übernommen.

8. mutuum 'Darlehen, Anleihe' gelihens : ein gelihens, das man entlechnet. Gelihens als Substantiv ist in der deutschen Wortgeschichte nicht belegt. Ohne Frage besteht eine etymologische Verbindung mit dem Partizip Perfekt (geliehen) des Verbs leihen. Adjektive und das Partizip Perfekt der Verben lassen sich als Substantive benutzen, vorausgesetzt, dass sie durch den Artikel das ergänzt werden. Die Tatsache, dass der Artikel bei Vrančić fehlt, kann die Wörterbuchbenutzer, die der deutschen Sprache wenig oder gar nicht mächtig sind, irreführen.

\footnotetext{
${ }^{2}$ Wegen Mangel an entsprechenden Buchstaben haben wir die Buchstaben des Originals durch $a e, u e, o e$ wiedergegeben. Im Original befindet sich über «a», «u» und «o» ein kleines «e».
} 
9. valvuli [valvolae] (P1.) 'Schale der Frucht der Hülsenpflanzen' kleine taedtle: die taedtlin in den Schotten oder schelffen des gemuess, in welchen ein jedes erbsslin oder boenlin besonder ligt. Taedtle ist in DWB nicht belegt. Vrančić modifizierte leicht das Substantiv von Calepinus.

\subsubsection{Ungarische Äquivalente}

1. cardus 'Distel' kóró ‘dürrer Stängel von Pflanzen' : bogács [bogáncs] kóró. Die Erklärung von Calepinus lässt sich entweder als zwei Wörter, 'Distel' bzw. 'dürrer Stängel von Pflanzen', oder als ein zusammengesetztes Wort bogá(n)cs kóró 'dürrer Distelstängel' interpretieren.

2. cubitus 'Ellenbogen' kar 'Arm' : könyék, kar. Vrančić übernahm das semantisch falsche Äquivalent.

3. ditio 'Macht, Gewalt, Botmäßigkeit', 'Provinz' : birodalom, tartomány. Auch das kroatische Äquivalent des lateinischen Stichwortes erweist sich als semantisch falsch: država 'Staat'.

4. genista 'Ginster' ganya fa bokor : ganya fa bokor. Es ist schwer, den Ausdruck zu rekonstruieren. Es ist die Interpretation gányafa, bokor möglich, aber auch die Sequenz gánya, fa, bokor ist nicht auszuschließen. Eine weitere Schwierigkeit liegt darin, dass sich der Ausdruck gányafa nicht interpretieren lässt.

5. ineptia 'Albernheiten, Geschwätz, Unsinn' alkolmatlanság 'Ungeeignetheit' : alkolmatlanság, apróság, germekeskedés. Alkolmatlanság kann in bestimmten Kontexten im Sinn von 'Albernheiten, Geschwätz, Unsinn' benutzt werden. Außerhalb dieser Kontexte verfügt aber alkolmatlanság nicht über diese erwähnte Bedeutung. Das Wörterbuch bot keinen Platz für Bemerkungen. Auch das kroatische Äquivalent von ineptia ist semantisch falsch: nepodobstvo 'Ungeeignetheit'.

6. instauratio 'Erneuerung' épités 'Bau' : föl épités, megújítás. In den Äquivalenten im Dictionarium fehlen häufig die Verbalpräfixe. Das beweist, dass Vrančić ihren Gebrauch wenig kannte. Bei Calepinus wird die Bedeutung des ersten Äquivalents durch das zweite präzisiert und nicht umgekehrt. Der von Vrančić übernommene erste Ausdruck kann ohne Kontext bzw. andere Bemerkungen nicht über die Bedeutung 'Erneuerung' verfügen.

7. Iyra 'Leier, Laute' hegedü 'Geiger' : hegedü (geschrieben als hegedw mit Strich über das «W»). Auch das deutsche und das kroatische Äquivalent, Harpfe [Harfe] bzw. guzle 'einsaitiges Streichinstrument', ist semantisch falsch.

8. maturitas 'Reife' erst : ertseg. Das Äquivalent von Vrančić scheint ein Druckfehler des Wortes bei Calepinus zu sein. Auch das Letztere ist eine fehlerhafte Form von érettség 'Reife'.

9. nefas 'Frevel, Gottlosigkeit, Greueltat, Sünde' iszonyú 'greulich' : iszonyú latorság 'greuliche Schurkerei'. Das bei Vrančić belegte Adjektiv, getrennt von seinem Substantiv, erweist sich als fehlerhaftes Äquivalent. Es ist nicht festzustellen, ob es sich um einen Druckfehler (dem Drucker könnte beim Setzen ein Wort hingefallen sein) oder um einen Kopierfehler von Vrančić handelt. Im zweiten Fall 
sollte Vrančić den ungarischen Ausdruck nicht als ein Lexem, sondern als eine Sequenz von zwei getrennten Wörtern interpretieren.

10. ruga 'Runzel' sömögözés : sömögözés. In den vor Calepinus entstandenen Wörterverzeichnissen bzw. Glossen entsprechen dem Wort ruga sömörgés (auch sömergés ist nicht auszuschließen, ZsélySzj.) und megsemergett orca (Murm.). Im letzten Beispiel wird das Adjektiv von einigen als megsömörgött interpretiert (RMG: 618). Die Äquivalente von rugosus 'faltig, runzelig' sind sömörgözött (GyönygySzt.), semergött (Murm.) (RMG: 618). Die Form sömögözés bei Calepinus ist offenbar fehlerhaft. Sie ist entweder ein Druck- bzw. Kopierfehler oder eine falsche Schreibweise des Adjektivs eines Ausdrucks Adjektiv + Substantiv. Auf jeden Fall wurde diese Form von Vrančić übernommen. Sein Äquivalent wurde durch RMG (618) als sömörgözés „,rekonstruiert“.

11. sagacitas '1. Spürkraft. 2. Scharfsinn, Scharfblick, Klugheit' fityézés : fityézés. Mundartliche Wörter wie fityerészik 'herumsuchen' (Naszva, Nordungarn), fityerész, fityerö (Adj.), 'lebhaft, flink, geschwätzig' (Fornos, heute Karpato-Ukraine) (ÚMTSz 2: 457) beweisen nicht die Existenz von fityézés bzw. dessen erwähnte Bedeutungen als semantische Äquivalente von sagacitas.

12. stipula 'Halm, bsd. Strohhalm' : szalmaköntö : szalma köntö. Die Interpretationsversuche der Äquivalente betonen nur deren fehlerhaften Charakter. Ein eventuelles Strohgewand oder das mundartliche köntö 'Rohrdecke für die Zudeckung des Bienenkorbs' (Kórógy, heute Kroatien) (ÚMTSz 3: 540) führen zu negativem Ergebnis.

13. vicia 'Wicke' vadbab (eigentl. 'Wildbohne') : rosnok, vad zab (eigentl. 'Wildhafer'). Das Äquivalent von Vrančić lässt sich entweder als ein Druckfehler des Ausdrucks bei Calepinus oder als eine fehlerhafte Lesart von $z a b$ interpretieren: vgl. das Minimalpaar zab 'Hafer' : $b a b$ 'Bohne'.

\subsubsection{Italienische und deutsche Äquivalente}

1. curculio 'Kornwurm' tignola, wirmblen, die da Koren est [isst] : tignuola, viblen, oder milwen die das korn fraessen und aushoelen. Die italienischen Äquivalente von curculio - gorgoglione, pinteruolo - sind seit dem 14. Jahrhundert bzw. seit 1476 belegt (DELIN: 680, 1286). Wirmblen 'Würmchen' ist ein Teil der Worterklärung. Kornwurm ist seit der mittelhochdeutschen Periode dokumentiert (DWB 5: 1832). Die Umschreibung des Lexems geht auf Calepinus zurück.

2. saga 'Wahrsagerin, Zauberin' incantatrice 'Zauberin', unhold 'Hexe' : incantatrice; ein haex, unhold, ein weib die mit zauberey umbgadt. Die italienischen bzw. deutschen Äquivalente von saga sind die Folgenden (Zeitpunkt der ersten Belege in Klammern): Wahrsagerin (mhd.), Zauberin (mhd.) (LGSWLD: 1016; DudenEt: 798, 825); maga (vor 1446), indovina (vor 1292) (DILL: 1397; DELIN: 912, 767). Auch die anderen Äquivalente von Vrančić erweisen sich als fehlerhaft: kroat. čarovnica [čarobnica], ung. boszorkány 'Hexe'. 


\subsubsection{Italienische, deutsche und ungarische Äquivalente}

caries 'Morschheit, Fäulnis' tarlo 'Zahnfäule', schemmel [Schimmel], óság : tarlo, Schimmel und fëule so von der elte eines dings kompt, szu etel, óság. Die Äquivalente von caries sind it. carie, intarlamento, corrosione (DILL: 193), ung. redvesség, korhadás, szuvasság (FLNySz: 308).

\subsection{Das Wörterbuch von Szikszai Fabricius}

1. pastinaca 'Pastinak' sárgarépa : sárgarépa 'Karote'.

2. pero 'roher Lederstiefel, bsd. der Bauern u. Soldaten' : bocskor 'Bundschuh' : bocskor.

\subsection{Das Wörterverzeichnis von Gábor Pesti}

1. oliva '1. Olive. 2. Ölbaum' olajmag ‘Ölsame' : olajmag. Dem Stichwort olea ‘1. Olive. 2. Ölbaum' bei Vrančić entsprechen it. oliva, dt. Olbaum [Ölbaum], kroat. maslina, ung. olajfa. Der Vergleich der Äquivalente der zwei gleichbedeutenden lateinischen Wörter weist darauf hin, dass Vrančić versuchte, die zwei Bedeutungen der lat. Stichwörter zu trennen und den Äquivalenten zuzuordnen. Für dieses Ziel übernahm er das Äquivalent von Calepinus in der Bedeutung 'Frucht des Ölbaums'.

2. tegmen 'Decke, Bedeckung, Hülle' lepecö : lepecö. Es handelt sich um einen Druckfehler von lepedö 'Betttuch'.

\subsection{Das Wörterbuch von Petrus Dasypodius}

1. cilium 'Augenwimper, bsd. die untere' Augbraw: augbrauw [Augenbraue]. Die Bedeutung des deutschen Wortes lässt sich mit Schwierigkeiten in der frühneuhochdeutschen Periode definieren. Im deutsch-lateinischen Wörterbuch von Dasypodius ist das Äquivalent von cilium aubgraw und das gleiche Wort - augbrauen 'beide Augenlider, Augenbraue' - kommt im Wörterbuch von Maaler vor (DWB 1: 788). Augbraw ist zweimal im deutsch-lateinischen Wörterbuch von Dasypodius belegt, das erste Mal als Äquivalent von cilium, das zweite Mal im Ausdruck die haar der augbawen (s. v. palpebrae 'Augenlid'). Diese Daten weisen darauf hin, dass Vrančić das Äquivalent von Dasypodius übernahm. Sowohl das kroatische als auch das ungarische Äquivalent von Vrančić ist falsch: kroat. obarve [obrve], ung. szemöldök 'Augenlid'.

2. salebrae '1. holperige Stelle. 2. (mittellat.) Risse, Sprünge (im Straßenpflaster)' Rauche weg: rauche/unaebne ort/odder wege. Bei der Interpretation des Ausdrucks in dieser Form entstehen Schwierigkeiten. Rauche [Rauhe, Räuhe] (DWB 8: 272) bzw. Weg lassen sich weder als zwei getrennte Wörter noch als Syntagma oder als ein zusammengesetztes Wort interpretieren. Es sind zwei Erklärungen möglich. Entweder befand sich im Manuskript der Ausdruck rauer Weg, der nicht richtig in der Druckerei gesetzt wurde, oder es ist die Quelle von Vrančić 
in Betracht zu ziehen. Meiner Meinung nach erweist sich die letztere Möglichkeit als wahrscheinlicher, da sie durch die Präsenz des Ausdrucks bei Dasypodius unterstützt wird. Doch die semantische Erklärung von Dasypodius wirft aus grammatischen Gesichtspunkten einige Interpretationsmöglichkeiten auf. Die ersten bzw. letzten Glieder des Ausdrucks - rauche wege - lassen sich als ein Lexem interpretieren, deren Komponenten in Pluralform stehen. Im Syntagma unaebne ort fehlt das Phonem /r/ im Auslaut. Das Zeichen / signalisiert, dass die Adjektive frei zu wählen sind und sich auf die Wörter Ort bzw. Wege beziehen. Beide Substantive sind im Nominativ; das erste im Singular, während das zweite im Plural steht. Diese Wortfolge konnte Vrančić irreführen, der die Ausdrücke rauche wege oder rauche weg wahrscheinlich seiner Quelle entnahm. Wenn im Manuskript das Wort wege gestanden hätte, konnte der Buchstabe «e» während des Satzes vor dem Druck entfallen.

3. sandalium 'Sandale' Pantofle : ein pantoffel. Das heutige deutsche Äquivalent ist in Pluralform seit dem 15. Jahrhundert belegt (DudenEt: 612).

4. situs 'Moder, Schimmel, Schmutz, Rost' Grauwe : Graewe/schimmel von feichtigkeyt/stinckechtig feytichgkeit.

5. solamen 'Trost' Trost, ergetzung : ein trost/ergetzung (s. v. solor). Die Bedeutung 'Trost' von Ergetzung ist in der deutschen Wortgeschichte nicht belegt.

6. tenor ' 1 . ununterbrochener Lauf, Schwung. Verlauf, Vortdauer, Zug, Zusammenhang' Weiß, Ordnung: der doa[!] der die rede weiset/die vollstreckung an eynander/ordnung. Die Bedeutungen von tenor lassen sich in der deutschen Wortgeschichte nicht dem Substantiv Ordnung zuschreiben. Das kroatische Äquivalent von tenor, red 'Ordnung, Reihe, Folge' ist ebenso semantisch fehlerhaft.

\subsection{Die Wörterbücher von Dasypodius und Calepinus}

Da die beiden Wörterbücher die gleichen negativen Übereinstimmungen aufweisen, ist es unmöglich zu bestimmen, welches von ihnen als Quelle benutzt wurde.

1. glarea 'Kies, grober Sand, bsd. Schotter' Ri $\beta$ : Riß/klein steinlin am gestad der flüssen/oder des meers (Dasypodius); Riss, sandechtig und griesechtig ardtrich, als gewonlich is tan den gestaden der wasseren (Calepinus). Es handelt sich um die semantisch falsche Anwendung des Substantivs Riese (mhd. rise) natürliche oder künstliche fläche an einem bergabhang, über die wasser, geröll oder holz herabfällt (DWB 8: 934).

2. palatum 'Gaumen' der rachen: der rache (Dasypodius); der rachen des mundts (Calepinus).

\subsection{Das Wörterverzeichnis von Ioannes Murmellius}

lyricen 'Leierspieler' hegedüs 'Geiger' : hegedes. Auch das deutsche bzw. kroatische Äquivalent ist semantisch falsch: Harpffenschlager [Harfenschläger], guzlar 'Spieler von guzle (eines einsaitigen Streichinstruments)'. 


\subsection{Das Wörterverzeichnis von Murmellius und das Wörterbuch von Calepinus}

planta 'Setzling, Absenker, Pfropfreis' ültetés 'Anbau, Anpflanzung' : oltovány, ültetés. Auch das kroatische Äquivalent, sad 'Anbau, Plantage', ist semantisch falsch. Die semantische Beziehung zwischen beiden Wörtern ist eindeutig.

\subsection{Das Wörterbuch von Hieronymus Megiser}

ciconia 'Storch' čaplja 'Reiher' : zhapla [čaplja].

\section{Zusammenfassung}

Die Äquivalente der angeführten lateinischen Stichwörter lassen Schlussfolgerungen in Bezug auf den Quellengebrauch von Vrančić zu.

Unser Autor hatte absolutes Vertrauen in seine Quellen. Diese Feststellung ist durch die große Anzahl der in unveränderter Form übernommenen Wörter bewiesen. Nur in einigen Fällen machen sich kleine Veränderungen bemerkbar: 1) bolzachino (s. v. cothurnus), 2) giemo (s. v. glomus), das unterstehen (s. v. conatus), kleine tadtle (s. v. valvuli), 5) építés (s. v. ineptia), 6) pantofflen (s. v. sandalium), 7) der rachen (s. v. palatum), 8) hegedüs (s. v. lyricen). Häufig wird nur die Schriftform der Wörter verändert $(1,2,3)$, ohne deren Aussprache zu modifizieren. Nur im Substantiv bolzachino wird die Aussprache infolge der Ersetzung der Buchstaben bzw. Phoneme /r/ $\rightarrow / 1 /$ verändert, was sich auch als Druckfehler interpretieren lässt. Die Umschreibung der Diminutivform taedtlin als kleine taedtle weist auf die Sprachkenntnisse von Vrančić hin. Auch die Veränderung hegedes $\rightarrow$ hegedüs beweist die Sprachkenntnisse unseres Autors.

Es ist nicht zu behaupten, dass Vrančić keines der Äquivalente in den benutzten Quellen kannte und seine Wortauswahl zufällig war. Seine Wahl wurde von verschiedenen Gesichtspunkten bestimmt.

Hinsichtlich der italienischen Äquivalente wurde bewiesen, dass sich Vrančić bewusst bemühte, die Latinismen, d. h. die ins phonomorphologische System des Italienischen integrierten Wörter, als mögliche Äquivalente zu vermeiden (die so genannten gelehrten Wörter, vgl. VIG 2006: 20). Dieses Bestreben wirkte auch im Fall der Wörter aggio (s. v. spatium) und dittione (s. v. vocabulum). Unter den Äquivalenten von spatium - spatio, aggio, tiempo - zog Vrančić das erste Äquivalent (spatio) als Latinismus nicht in Betracht. Spazio ist in den heutigen lateinischitalienischen Wörterbüchern als Äquivalent belegt. Er beachtete tiempo nicht, weil ihm bekannt war, dass dieses Wort keine italienische Form ist. Aus dem kurzen lat. /ě/ wurde in offener Silbe auf Italienisch / $\varepsilon /$ und kein Diphthong. Aber er musste auch bemerken, dass die Bedeutung von tempo 'Zeit' zu allgemein ist. Es blieb ihm keine andere Wahl, als das ihm unbekannte aggio zu übernehmen. Aus ähnlichen Gründen schließt er auf das in seiner Form latinisierende Wort vocabulo (heute vocabolo). Vermutlich war ihm die Bedeutung 'Wort' (der erster Beleg vor 
1321, DELIN: 1829) des Substantivs voce 'Stimme' unbekannt, so blieb ihm nur dittione, das auch ein Latinismus ist.

Auch zwei andere Typen des Quellengebrauchs von Vrančić beruhen auf der Interpretation der Äquivalente seiner Quellen.

Vrančić verstand die Umschreibungen mindestens teilweise, sonst konnte er nicht entscheiden, welche Elemente er übernehmen und welche er weglassen sollte. Die so übernommenen Wörter erwiesen sich als semantisch unpräzise Äquivalente.

In den Fällen, in denen sich mehrere Äquivalente in den Quellen befanden, wählte Vrančić die Wörter nicht instinktiv aus. Wenn es so gewesen wäre, hätte er sich immer für das erste bzw. kürzeste Äquivalent entschieden. Sein Verfahren wurde mit Sicherheit davon bestimmt, dass er das ausgewählte Wort in mindestens einer Bedeutung kannte. Teilweise waren ihm auch die anderen Lexeme bekannt. So versuchte er das ,richtige“ Äquivalent auszuwählen.

Es wurde bewiesen, dass Vrančić sechs Wörterbücher benutzte. Es wurde die Meinung einiger Forscher (Melich, Balázs) bestätigt, dass unser Autor Calepinus konsultierte. Außerdem haben wir auch zahlreiche neue Belege ans Licht gebracht.

Auf Grund der negativen Übereinstimmungen erhöhte sich die Anzahl der von Calepinus übernommenen Wörter.

Eine andere Neuheit besteht darin, dass Calepinus die Quelle eines Teils der italienischen und deutschen Lexeme ist. Hinsichtlich der deutschen Wörter richtete die Forschung, außer Žepić, keine Aufmerksamkeit auf deren Quellen.

Eine andere wichtige Quelle der deutschen Lexeme ist das Wörterbuch von Petrus Dasypodius. Theoretisch ist nicht auszuschließen, dass die Wörter, die auf Calepinus bzw. Dasypodius als Quellen hinweisen, einem dritten zwei- oder mehrsprachigen Wörterbuch entstammen. Aber eine derartige Annahme ist noch nicht bewiesen. Diese Bemerkung gilt auch für die italienischen Lexeme, die nach unserem heutigen Wissensstand von Calepinus übernommen wurden.

Der Gebrauch von drei weiteren Quellen (Murmellius, Szikszai Fabricius, Gábor Pesti) wurde bestätigt. Ihre Benutzung ist aus mehreren Aspekten beachtenswert. Der allgemeinen Meinung nach benutzte Szikszai Fabricius die Werke von Murmellius und anderen Autoren (RMG: 45). Die Belege von RMG zeigen ein autonomes Verfahren von Szikszai Fabricius, der seiner Quelle nicht kritiklos folgte. Seine semantisch falschen Äquivalente, die Vrančić übernahm, sind ein Beweis seiner Autonomie bei der Verfassung seines Wörterbuchs. Die bisherige Meinung der ungarischen Lexikografie, nach der das Wörterverzeichnis von Gábor Pesti in erster Linie im Ausland bekannt war, wird entkräftet (BÁRCZI-BENKÖBERRÁr 1980: 60). Diese Meinung beinhaltet implizit, dass Gábor Pesti keinen Einfluss auf die Lexikografie in Ungarn ausübte. Die erwähnten Beweise widersprechen dieser These.

Es ist auffällig, dass die Gesamtzahl der Lexeme aus den Quellen im Vergleich zur Anzahl der semantisch falschen Wörter geringer ist, als die Menge der nicht belegten bzw. deformierten Äquivalente. Diese Tatsache ermöglicht, Schlussfolgerungen in Bezug auf die Verfassung des Dictionariums zu ziehen. 
Es wurde schon erwähnt, dass Vrančić bei der Verfassung der lateinischen Stichwörter seine eigenen Wortkenntnisse aktivierte, ohne Calepinus dafür zu benutzen. Er schrieb die Äquivalente der einzelnen Sprachen autonom auf Grund seiner Kenntnisse ins Manuskript. Im Fall von Schwierigkeiten schreckte er vor individuellen Wortbildungen bzw. Umschreibungen nicht zurück. Er war gezwungen, weil er das Dictionarium lange Zeit und mit Unterbrechungen verfasste und nicht immer Zugang zu Wörterbüchern hatte. Hinsichtlich der objektiven Möglichkeiten der Epoche - es war nicht leicht, Bücher wegen ihrem hohen Preis zu kaufen, öffentliche Bibliotheken existierten praktisch nicht - ist festzustellen, dass Vrančić bestimmte Quellen gelegentlich benutzte, wenn er zu ihnen Zugang hatte. Dies erklärt die relativ hohe Anzahl seiner Quellen und die relativ kleine Anzahl der übernommenen Lexeme. Es ist zu vermuten, dass er die Belege der Quellen, die ihm gelegentlich zur Verfügung standen, in den frei gelassenen Platz seines Manuskriptes einfügte. Die Quellen waren mit großer Wahrscheinlichkeit im Besitz seiner Bekannten und Freunde. Vrančić lieh die Quelle für kurze Zeit von ihnen aus oder benutzte sie bei einem Besuch.

\section{Literatur}

AIS = JABERG Karl, JUD Jakob: Sprach- und Sachatlas Italiens und der Südschweiz. I-VIII. Zofingen, 1928-1940.

BALÁzS 1975 = BALÁzS János: L’importanza del «Dictionarium» (1595) e della «Logica Nova» (1616) di Faustus Verancsics, pubblicati a Venezia. In: KLANICZAY Tibor (ed.): Rapporti veneto-ungheresi all'epoca del Rinascimento. Budapest, 1975. 373-388.

BÁRCZI 1960 = BÁRCZI Géza: Eltérések Verancsics Faustus szótára 1595-i kiadásainak különféle példányai között. Magyar Könyvszemle 76 (1960): 29-32.

BÁrCZI-BENKÖ-BERRÁr 1980 = BÁrcZi Géza, BenKö Loránd, Berrár Jolán: A magyar nyelv története. Budapest, ${ }^{3} 1980$.

BETHLEN 1981 = Bethlen Mihály útinaplója [1691-1695]. Sajtó alá rendezte és az utószót írta Jankovics József. Budapest, 1981.

CAlepinus 1585 = Calepinus Ambrosius: Dictionarium decem linguarum. Lat., Hebr., Graec., It., Germ., Hisp., Pol., Ung., Angl. Lugduni, 1585.

Calepinus 1586 = Calepinus Ambrosius: Dictionarium decem linguarum. Lat., Hebr., Graec., It., Germ., Hisp., Pol., Ung., Angl. Lugduni, 1586.

DDF $=$ SAMANI Salvatore: Dizionario del dialetto fiumano. Venezia, 1980.

DDV = BOERIo Giuseppe: Dizionario del dialetto veneziano [Venezia, 1856]. Ristampa anastatica. Firenze, 1983.

De SMET 1974 = De SMET Gilbert: Einführung. In: DAsypodius Petrus: Dictionarium latinogermanicum. Mit einer Einführung von Gilbert de Smet [Straßburg, 1536]. Hildesheim-New York, 1974.

DELIN = CORTELAZZO Manlio, Zolli Paolo: Dizionario etimologico della lingua italiana . Seconda ediz. in volume unico. Bologna, 1999.

DILL = BIANCHI Enrico, BIANCHI Raffaello, LeLli Onorio: Dizionario illustrato della lingua latina. Firenze, ${ }^{2} 1974$.

DLG = DASYPODIUS Petrus: Dictionarium latinogermanicum. Mit einer Einführung von Gilbert de Smet [Straßburg, 1536]. Hildesheim-New York, 1974. 
DSFEDC = NANZINI Giulio, Rocchi Luciano: Dizionario storico fraseologico etimologico del dialetto di Capodistria. Trieste-Rovigno, 1995.

Du CANGE = Du Fresne Du CANGE Charles: Glossarium mediae et infimae latinitatis I-V. Nachdruck der Ausgabe von 1883-1887. Graz, 1954.

DudenEt $=$ Duden Etymologie. Herkunftswörterbuch der deutschen Sprache. Bearbeitet von Günther Drosdowski. Mannheim-Leipzig-Wien-Zürich, ${ }^{2} 1997$.

DuKAT 1925 = DuKAT Vladoje: Rječnik Fausta Vrančića. Rad JAZU 231. Zagreb, 1925. $102-136$.

DWB = GRIMM Jacob, GRIMM Wilhelm: Deutsches Wörterbuch I-XVI. Leipzig, 18541971.

EV $=$ Prati Angelico: Etimologie venete. Venezia-Roma, 1968.

FISCHER 1904-1914 = FISCHER Hermann: Schwäbisches Wörterbuch I-IV. Tübingen, 19041914.

FLNySz = FINÁLY Henrik: A latin nyelv szótára [1884]. Reprint. Budapest, 2002.

FNHD = GöTZE Alfred: Frühneuhochdeutsches Glossar. Berlin, ${ }^{7} 1967$.

FOKOS 1924 = FoKos Dávid: Szótárirodalmunk történetéhez. Magyar Nyelvőr 53 (1924): 74-76.

GDDT = Doria Mario: Grande dizionario del dialetto triestino. Storico etimologico fraseologico. Con la collaborazione di Claudio Noliani. Trieste, 1987.

GDLI = BATTAGLIA Sandro (red.): Grande dizionario della lingua italiana I-XXI. Torino, 1961-2002.

GMILRH = BARTAL Antal: Glossarium mediae et infimae latinitatis regni Hungariae [Lipsia, 1901]. Budapest, 1983.

GYLM = GYÖRKÖSY Alajos: Latin-magyar szótár. Budapest, ${ }^{5} 1975$.

GYÖNGYÖSI 1998 = GYÖNGYÖSI István: Mással társalkodó Murányi Vénus. Budapest, 1998.

GYÖNGYÖSI 1999 = GYÖNGYÖsI István: Porából megéledett Fönix. Budapest, 1999.

GYÖNGYÖSI 2000 = GYÖNGYÖSI István: Thököly Imre és Zrínyi Ilona házassága. Budapest, 2000.

GYÖNGYÖSI 2002 = GYÖNGYÖSI István: Rózsakoszorú. Budapest, 2002.

GYÖNGYÖSI 2003 = GYÖNGYÖSI István: Csalárd cupido. Proserpina elragadtatása. Cuma városában épittetett Dádalus temploma. Herolda fordítások. Budapest, 2003.

Heltai 1981 = Heltai Gáspár: Krónika az magyaroknak dolgairól. Sajtó alá rendezte Kulcsár Margit. A bevezetőt Kulcsár Péter írta. Budapest, 1981.

HERBERT 1976 = HERBERT Jean (szerk.): Konferenciaszótár. Küldöttek és tolmácsok kézikönyve. Angol, francia, spanyol, orosz, olasz, német. Magyar függelékkel. 2., átdolgozott és bővített kiadás. Budapest, 1976.

IsTVÁNFFY 2001 = Istvánffy Miklós magyarok dolgairól irt krónikája Tállyay Pál XVII. századi forditásában. I/1-2. Budapest, 2001.

JAKAB-BÖLCSKEI 2000 = JAKAB László, BÖLCSKEI András: Balassi-szótár. Debrecen, 2000.

LGSWLD = PERTSCH Erich: Langenscheidts Großes Schulwörterbuch. Lateinisch-Deutsch. Berlin-München-Wien-Zürich, 1977.

LLMAH = BORONKAI Iván (szerk.): Lexicon latinitatis medii aevi Hungariae I-. Budapest, 1987-.

LLMAI = KostrenČIĆ Marko, GorTAN Veljko, Herkov Zlatko (ed.): Lexicon latinitatis medii aevi Jugoslaviae I-II. Zagreb, 1973.

LLNMA = FuchS Johanne W., WIEGERS Olga, GUMPERT-HEPP Marijke: Lexicon Latinitatis Nederlandicae medii aevi. Wordenboek van het middeleuws latijn van de Noordelijske Nederlanden I-VIII. Amsterdam-Leiden-Boston, 1970-2003. 
LMALB $=$ VAREL Ladislav, MARTínEK Jan (ed.): Latinitatis medii aevi Lexicon bohemorum. Slovník středověké latiny v českých zemích. I-. Praha, 1987-.

LMILP = PLEZIA Mariana (ed.): Lexicon mediae et infimae latinitatis polonorum - Stownik laciny średniowiecznej $v$ Polsce. I-. Wrocław-Kraków-Warszawa, 1953-.

MEGISER 1967 = MEGISER Hieronymus: Slovenisch-deutsch-lateinisches Wörterbuch. Neugestaltung und Faksimile der ersten Ausgabe aus dem Jahr 1592. Bearb. von Annelies Lägreid. Wiesbaden, 1967.

Melich 1906 = Melich János: A magyar szótárirodalom (Negyedik közlemény). Nyelvtudományi Közlemények 36 (1906): 165-206.

Melich 1913 = Melich János: Adalékok a magyar szótárirodalomhoz. Nyelvtudományi Közlemények 43 (1913): 242-244.

MHDHWB = LEXER Mathias: Mittelhochdeutsches Handwörterbuch I-III. [Leipzig, 18721878.] Stuttgart, 1979.

Murmellius 1533 = Lexicon Ioannis Murmellii, in quo Latina rerum uocabula in suas singula digesta classes, cum Germanica et Hungarica interpretatione. Cracouia, 1533.

NySz = SzARVAS Gábor, SimONYI Zsigmond: Magyar nyelvtörténeti szótár a legrégibb nyelvemlékektöl a nyelvújitásig I-III. Budapest, 1890-1893.

OklSz = Szamota István, Zoltai Gyula: Magyar oklevél-szótár. Pótlék a magyar nyelvtörténeti szótárhoz. [Budapest, 1902-1906.] Budapest, 1984.

Pesti 1975 = Nomenclatvra sex Lingvarvm, Latinae, Italicae, Gallicae, Boemicae, Hungaricae et Germanicae [...] per Gabrielem Pannoniu(m) Pesthinum... [Wien, 1538.] Budapest, 1975.

RMG = BERRÁr Jolán, KÁROLy Sándor (szerk.): Régi magyar glosszárium. Szótárak, szójegyzékek és glosszák egyesitett szótára. Budapest, 1984.

SULYOK 2001 = SULYOK Hedvig: Magyar-olasz és olasz-magyar szójegyzék Verancsics Faustus Dictionariuma alapján. Lessico ungherese-italiano italiano-ungherese in base al Dictionarium di Faustus Verantius. Szeged, 2001.

SzEnCI MolnÁr 1976 = Szenci Molnár Albert válogatott müvei. Tolnai Gábor irányításával sajtó alá rendezte Vásárhelyi Judit. A bevezetést írta Tolnai Gábor. Budapest, 1976.

SzIKSZAI FABRICIUS 1590 = SzIKSZAI FABRICIUS Balázs: Nomenclatvra sev Dictionarivm Latino-vngaricum. Debrecini, 1590.

SzT = SzABÓ T. Attila (szerk.): Erdélyi magyar szótörténeti tár I-XIV. Bukarest-Budapest-Kolozsvár, 1976-2014.

TESz = BENKŐ Loránd (szerk.): A magyar nyelv történeti-etimológiai szótára I-IV. Budapest, 1967-1984.

ÚMTSz = B. LÖRINCZY Éva, HosszÚ Ferenc (szerk.): Új magyar tájszótár I-V. Budapest, 1979-2010.

VDCh = NACCARI Riccardo, Boscolo Giorgio: Vocabolario del dialetto chiogiotto. Chioggia, 1982.

VDVD = Miотто Luigi: Vocabolario del dialetto veneto-dalmata. Trieste, 1982.

VFDB = DOMINI Silvio, FUlizIO Aldo, Miniussi Aldo, VitTORI Giordano: Vocabolario fraseologico del dialetto "bisiàc". Bologna, 1985.

$\mathrm{VG}=$ RoSAMANI Enrico: Vocabolario giuliano. Bologna, 1958.

VIG 2005 = VIG István: Kroatizmi u četverojezičnom rječniku Hieronyma Megisera. In: LUKAČ Stjepan (ured.): Na brzu ruku skupljeni skup. Budapest, 2005. 265-276.

VIG 2006 = VIG István: Equivalenti italiani di termini specifici latini in alcuni dizionari plurilingue del '500. In: TegYey Gabriella, DéSfalvi Tóth András, A. MihÁLyKa Lívia (ed.): Écritures. Scritture. Actes du colloque. Veszprém, 2006. 11-22. 
VIG 2012 = VIG István: Zuverlässigkeit älterer mehrsprachiger Wörterbücher am Beispiel des fünfsprachigen Dictionariums von Faust Vrančić. Studia Slavica Hung. 57 (2012): $253-311$.

VIG 2014 = VIG István: Die Sprachkenntnisse von Fraust Vrančić im Licht seines fünfsprachigen Dictionariums. Studia Slavica Hung. 59 (2014): 239-290.

VONČINA 1979 = VoNČINA Josip: Vrančićev rječnik. Filologija 9 (1979): 7-36.

VRANČIĆ 1992 = VRANČIĆ Faust: Dictionarium quinque nobilissimarum Europae linguarum. Latinae, Italicae, Germanicae, Dalmatie [sic!] \& Ungaricae [Venezia, 1595]. Drugo bibliofilsko izdanje. Zagreb, 1992.

ŽEPIĆ 1992-1993 = ŽEPIĆ Stanko: Njemački vokabular u Vrančićevu Dikcionaru. In: Filologija 1992-1993 (20-21): 573-583. 